\title{
Exponentially Small Adiabatic Invariant for the Schrödinger Equation*
}

\author{
A. Joye ${ }^{1}$ and Ch-Ed. Pfister ${ }^{2}$ \\ 1 Département de Physique, Ecole Polytechnique Fédérale, CH-1015 Lausanne, Switzerland \\ 2 Département de Mathématiques, Ecole Polytechnique Fédérale, CH-1015 Lausanne, \\ Switzerland
}

Received July 7, 1990; in revised form December 1, 1990

Abstract. We study an adiabatic invariant for the time-dependent Schrödinger equation which gives the transition probability across a gap from time $t^{\prime}$ to time $t$. When the hamiltonian depends analytically on time, and $t^{\prime}=-\infty, t=+\infty$ we give sufficient conditions so that this adiabatic invariant tends to zero exponentially fast in the adiabatic limit.

\section{Introduction}

Let $H(t), t \in \mathbb{R}$, be a self-adjoint operator on a Hilbert space $\mathscr{H}$. We study the time-dependent Schrödinger equation in the adiabatic limit, i.e.

$$
i \varepsilon \frac{\partial}{\partial t} \varphi(t)=H(t) \varphi(t), \quad t \in \mathbb{R}
$$

when $\varepsilon \rightarrow 0$. The self-adjoint operator $H(t)$ satisfies three conditions.

I. Self-Adjointness and Analyticity. There exists a band $S_{a}$ in the complex plane, $S_{a}=\{t+i s:|s|<a\}$, and a dense domain $D \subset \mathscr{H}$ such that for each $z \in S_{a}, H(z)$ is a closed operator defined on $D, H(z) \varphi$ is holomorphic on $S_{a}$ for each $\varphi \in D$ and $H(z)^{*}=H(\bar{z})$. Moreover we suppose that $H(t)$ is bounded from below for $t \in \mathbb{R}$.

II. Behaviour at Infinity. There exist two self-adjoint operators $\mathrm{H}^{+}$and $\mathrm{H}^{-}$, bounded from below and defined on $D$, two positive constants $C$ and $\alpha$ such that for all $\varphi \in D$ and $|t|$ large enough

$$
\sup _{|s|<a}\left\|\left(H(t+i s)-H^{+}\right) \varphi\right\| \leqq \frac{C}{(1+|t|)^{1+\alpha}}\left(\|\varphi\|+\left\|H^{+} \varphi\right\|\right), \quad t>0
$$

\footnotetext{
* Supported by Fonds National Suisse de la Recherche, Grant 2000-5.600
} 
and

$$
\sup _{|s|<a}\left\|\left(H(t+i s)-H^{-}\right) \varphi\right\| \leqq \frac{C}{(1+|t|)^{1+\alpha}}\left(\|\varphi\|+\left\|H^{-} \varphi\right\|\right), \quad t<0 .
$$

III. Separation of the Spectrum. There exist two $C^{1}$-functions $e_{1}(t)$ and $e_{2}(t)$ and a positive constant $\delta^{*}$ such that for all $t \in \mathbb{R}, e_{2}(t)-e_{1}(t) \geqq \delta^{*}$ and the closed interval $\left[e_{1}(t), e_{2}(t)\right]$ belongs to the resolvent set of $H(t)$. We also suppose that $\lim _{t \rightarrow \pm \infty} e_{i}(t)=e_{i}^{ \pm}, i=1,2$ and $|t|^{1+\alpha}\left|e_{i}(t)-e_{i}^{ \pm}\right|,|t|^{1+\alpha}\left|e_{i}^{\prime}(t)\right|$, for $i=1,2$ are uniformly bounded on $\mathbb{R}$.

Condition III implies that the spectrum $\sigma(t)$ of $H(t)$ is separated into two parts $\sigma_{1}(t)$ and $\sigma_{2}(t)$ such that $\sigma_{1}(t) \subset\left(-\infty, e_{1}(t)\right)$ and $\sigma_{2}(t) \subset\left(e_{2}(t), \infty\right)$. By choosing the width of the band small enough, we can assume that the spectrum of $H(z)$ is also separated into $\sigma_{1}(z)$ and $\sigma_{2}(z), \sigma_{1}(z)$ being a bounded subset. Let $P_{1}(z)$ and $P_{2}(z)$ be the corresponding spectral projectors. These projectors provide a smooth decomposition of the Hilbert space

$$
\mathscr{H}=P_{1}(z) \mathscr{H} \oplus P_{2}(z) \mathscr{H} \equiv \mathscr{H}_{1}(z) \oplus \mathscr{H}_{2}(z)
$$

$\left(\mathscr{H}_{1}(z)\right.$ is not orthogonal to $\mathscr{H}_{2}(z)$ if $\left.z \notin \mathbb{R}\right)$.

If we put $t=\varepsilon s$ then Eq. (1.1) is equivalent to

$$
i \frac{\partial}{\partial s} \psi(s)=H(\varepsilon s) \psi(s)
$$

and thus we are considering a time-dependent Quantum Mechanical system with a slowly varying Hamiltonian. A very simple but important example is a spin-1/2 in a slowly varying time-dependent magnetic field. In that case $H(s)$ is simply a $2 \times 2$ self-adjoint traceless matrix. Condition III means here that the two eigenvalues of $H(s)$ do not cross. We can also think of the family $H(t), t \in \mathbb{R}$ as a smooth interpolating family of Hamiltonians between $H(-)$ and $H(+)$. For example $H(-)=H_{0}$, a self-adjoint operator, and $H(+)=H_{0}+V$ with $V$ a symmetric operator which is $H_{0}$-bounded with $H_{0}$-bound smaller than 1 . In this case we can choose

$$
H(t)=H_{0}+\frac{1}{2}(\tanh t+1) V .
$$

The main purpose of the paper is to study the following problem. Let $\varphi_{\varepsilon}(t)$ be a normalized solution of

$$
i \varepsilon \frac{\partial}{\partial t} \varphi(t)=H(t) \varphi(t), \quad \varphi\left(t^{\prime}\right)=\varphi^{*} \in D
$$

We choose the initial condition $\varphi^{*} \in D \cap \mathscr{H}_{1}\left(t^{\prime}\right)$ and we estimate the probability $\left\|P_{2}(t) \varphi_{\varepsilon}(t)\right\|^{2}$ to find the system in the spectral subspace $\mathscr{H}_{2}(t)$ by a measurement made at time $t$. It is convenient to introduce

$$
\begin{aligned}
\mathscr{P}_{21}\left(t, t^{\prime}\right)= & \sup \left\{\left\|P_{2}(t) \varphi_{\varepsilon}(t)\right\|^{2} \mid \varphi_{\varepsilon}(\cdot) \text { is a solution of }(1.1)\right. \\
& \text { with } \left.\left\|\varphi_{\varepsilon}\left(t^{\prime}\right)\right\|=1 \text { and }\left\|P_{1}\left(t^{\prime}\right) \varphi_{\varepsilon}\left(t^{\prime}\right)\right\|=1\right\} .
\end{aligned}
$$

The Adiabatic Theorem of Quantum Mechanics implies that

$$
\mathscr{P}_{21}\left(t, t^{\prime}\right)=O\left(\varepsilon^{2}\right)
$$


uniformly in $t$ and $t^{\prime}$ (see Theorem (3.1)). (To prove this result it is sufficient that $H(t)$ is strongly $C^{2}$ on $D$ and that $\frac{d^{k}}{d t^{k}} H(t)$ tends to zero at infinity as in condition III for $k=1,2$.) Result $(1.7)$ shows that $\mathscr{P}_{21}\left(t, t^{\prime}\right)$ is an adiabatic invariant for the Eq. (1.1). If $H(t)$ is analytic we can prove a much stronger result when we take the limits $t^{\prime} \rightarrow-\infty$ and $t \rightarrow \infty$. Let

$$
\begin{aligned}
\mathscr{P}_{21}(+\infty,-\infty)= & \sup \left\{\lim _{t \rightarrow \infty}\left\|P_{2}(t) \varphi_{\varepsilon}(t)\right\|^{2} \mid \varphi_{\varepsilon}(\cdot) \text { is a solution of }(1.1)\right. \\
& \text { with } \left.\left\|\varphi_{\varepsilon}(t)\right\|=1 \text { and } \lim _{t \rightarrow-\infty}\left\|P_{1}(t) \varphi_{\varepsilon}(t)\right\|=1\right\} .
\end{aligned}
$$

Theorem 1.1. Let $H(z)$ be an operator satisfying conditions $I, I I$ and $I I I$. Then there exist positive constants $\kappa$ and $M$ such that for small enough $\varepsilon$

$$
\mathscr{P}_{21}(+\infty,-\infty) \leqq \varepsilon^{2} M \exp \left(-\frac{2 \kappa\left(e_{2}(\infty)-e_{1}(\infty)\right)}{\varepsilon}\right)
$$

$A$ similar result holds if we exchange the role of $P_{1}$ and $P_{2}$ :

$$
\mathscr{P}_{12}(+\infty,-\infty) \leqq \varepsilon^{2} M \exp \left(-\frac{2 \kappa\left(e_{2}(\infty)-e_{1}(\infty)\right)}{\varepsilon}\right)
$$

Remarks.

i) The constant $\kappa$ depends in particular on the choice of the functions $e_{i}(t)$. The distance between $e_{1}(t)$ and $\sigma_{1}(t)$ is larger than some positive $\delta_{1}^{*}$ for all $t$. Similarly $e_{2}(t)$ is at a distance at least $\delta_{2}^{*}$ from $\sigma_{2}(t)$. If we decrease $\delta_{1}^{*}$ and $\delta_{2}^{*}$, then we can increase $e_{2}(\infty)-e_{1}(\infty)$. However $\kappa$ decreases.

ii) It is essential that we take the limits $t \rightarrow \pm \infty$, otherwise the theorem is not true. The analyticity property is also essential, at least in our proof, since we use a complex time. Notice that all derivatives of $\boldsymbol{P}_{k}(t)$ vanish at infinity as a consequence of the analyticity and decay conditions.

iii) In Classical Mechanics there is a well-known problem which is to estimate the variation of the adiabatic invariant $\Delta I=I(+\infty)-I(-\infty)$ of an oscillator

$$
\frac{d^{2}}{d t^{2}} x(t)=-\omega^{2}(\varepsilon t) x(t)
$$

when $\omega(-\infty)=\omega_{-}$and $\omega(+\infty)=\omega_{+}$. If $\omega$ is an analytic function which is strictly positive on the real axis and behaves reasonably at infinity then $\Delta I$ is exponentially small in $\varepsilon$ (see [1] Sect. 20). This problem and our problem for the case where $H(t)$ is a $2 \times 2$ matrix are very similar. In particular the positivity of $\omega$ corresponds to our condition III.

iv) There are many papers treating the case of a spin-1/2 in a time-dependent magnetic field since it is a case of considerable interest in physics. However there are very few mathematical results. It is indeed notoriously difficult to prove the validity of such exponentially small corrections in singular perturbation problems. An important paper in this direction is [2]. Only recently a proof of Theorem (1.1) has been given for the case of $n \times n$ matrices [3] and [4]. After the completion of this work we received a paper on the same subject [5]. The results are weaker. 
Essentially only bounded operators with discrete spectrum are treated whereas we have no condition on the nature of the spectra $\sigma_{1}(t)$ and $\sigma_{2}(t)$. Moreover the authors must introduce a very strong condition in order to obtain an exponential bound as in Theorem (1.1), so that their results do not even cover the case of $2 \times 2$ matrices. The reason for that is that they do not have our notion of dissipative paths (see below).

The paper is organized as follows. We prove the theorem for bounded operators in Sect. (4) by taking a complex time, and then for unbounded operators in Sect. (6) by approximating the unbounded operators by bounded ones. In the proof of Sect. (4) we introduce the notion of dissipative paths in the complex plane. Only along such paths we can get useful bounds for the evolution. The existence of such dissipative paths is discussed in Sect. (5) and the ideas of this section may be interesting in a broader context. It is crucial that all bounds depend only on the parameters appearing in conditions II and III and in particular the results of Sects. (4) and (5) must be independent of the norms of the operators. We have collected some basic estimates in Sect. (2) and recalled the notion of adiabatic evolution in Sect. (3).

\section{Basic Estimates}

Throughout this paper $\mathfrak{R} z$ is the real part of $z, \mathfrak{I} z$ is the imaginary part of $z$ and 1 denotes the identity operator.

Let $z \in S_{a}$. If $\lambda \in \rho(z)$, the resolvent set of $H(z)$, then $R(z, \lambda)=(H(z)-\lambda)^{-1}$. Similarly we define $R(+, \lambda)=\left(H^{+}-\lambda\right)^{-1}$ and $R(-, \lambda)=\left(H^{-}-\lambda\right)^{-1}$. Since $H(z)$ is closed, the domain $D$ with the the norm

$$
\|\varphi\|_{z}=\|\varphi\|+\|H(z) \varphi\|
$$

is a Banach space. The same is true for the norms

$$
\|\varphi\|_{ \pm}=\|\varphi\|+\left\|H^{ \pm} \varphi\right\| .
$$

By the closed graph theorem any two of these norms are equivalent. Let $X_{z}$, respectively $X_{ \pm}$, be the Banach space $D$ with the norm $\|\cdot\|_{z}$, respectively $\|\cdot\|_{ \pm}$. The function $z \mapsto H(z)$ is a holomorphic map on $S_{a}$ with values in $\mathscr{L}\left(X_{z}, \mathscr{H}\right)$ or $\mathscr{L}\left(X_{ \pm}, \mathscr{H}\right)$. The norms in these spaces are denoted by $\|\cdot\|_{z}$ or $\|\cdot\|_{ \pm}$. For any $\varphi \in D$ we define the operator $H^{(n)}(z)$ by

$$
H^{(n)} \varphi:=\frac{d^{(n)}}{d z^{(n)}}(H(z) \varphi) .
$$

When $z \in \mathbb{R}$ this operator is symmetric. We can express $H^{(n)}, n \geqq 0$, by the Cauchy formula

$$
H^{(n)}=\frac{n !}{2 \pi i} \oint_{\gamma} \frac{H\left(z^{\prime}\right) d z^{\prime}}{\left(z^{\prime}-z\right)^{n+1}},
$$

where $\gamma$ is a simple closed path in $S_{a}$ around $z$. The orientation of $\gamma$ is counterclockwise. All closed paths in the paper will have this orientation.

Let $z \in S_{a}$ and $\Omega$ be a convex compact subset in $S_{a}$. Then there exists a constant 
$M(z)$ such that for any $\varphi \in D$ and $z^{\prime}$ in $\Omega$,

$$
\left\|H^{\prime}\left(z^{\prime}\right) \varphi\right\| \leqq M(z)\|\varphi\|_{z}
$$

Therefore, if $z_{1}$ and $z_{2} \in \Omega$,

$$
\left\|\left(H\left(z_{1}\right)-H\left(z_{2}\right)\right) \varphi\right\| \leqq M(z)\left|z_{1}-z_{2}\right|\|\varphi\|_{z} .
$$

Let us choose $z=z_{1}$. Then for any $z_{2} \in \Omega$ such that $\left|z_{2}-z_{1}\right|$ is small enough,

$$
\frac{1}{1+M\left(z_{1}\right)\left|z_{1}-z_{2}\right|}\|\varphi\|_{z_{2}} \leqq\|\varphi\|_{z_{1}} \leqq \frac{1}{1-M\left(z_{1}\right)\left|z_{1}-z_{2}\right|}\|\varphi\|_{z_{2}} .
$$

From (2.7) we prove that $\|\cdot\|_{z}$ is continuous in $z$. Indeed, if $A \in \mathscr{L}\left(X_{z_{2}}, \mathscr{H}\right)$ then

$$
\|A \varphi\| \leqq\|A\|_{z_{2}}\|\varphi\|_{z_{2}} \leqq\|A\|_{z_{2}}\left(1+M\left(z_{1}\right)\left|z_{1}-z_{2}\right|\right)\|\varphi\|_{z_{1}} .
$$

Thus we get from (2.8) an upper bound for $\|A\|_{z_{1}}$. In a similar way we derive a lower bound. We have

$$
\left|\|A\|_{z_{2}}\left(1-M\left(z_{1}\right)\left|z_{1}-z_{2}\right|\right) \leqq\|A\|_{z_{1}} \leqq\|A \mid\|_{z_{2}}\left(1+M\left(z_{1}\right)\left|z_{1}-z_{2}\right|\right) .\right.
$$

Using the estimate (2.9) we prove that the function $\left(z, z^{\prime}\right) \mapsto\|H(z)\| \|_{z^{\prime}}$ is continuous. Let $\Omega$ be any compact subset of $S_{a}$. Then

$$
\sup _{\varphi \in D} \sup _{z_{1}, z_{2} \in \Omega} \frac{\left\|H\left(z_{1}\right) \varphi\right\|}{\|\varphi\|_{z_{2}}}=\sup _{z_{1}, z_{2} \in \Omega}\left\|H\left(z_{1}\right)\right\|_{z_{2}} \leqq K<\infty
$$

and

$$
\|\varphi\|_{z_{1}} \leqq(K+1)\|\varphi\|_{z_{2}}, \quad z_{1}, z_{2} \in \Omega .
$$

On the other hand, using condition II, we can compare any norm $\|\varphi\|_{z}$ with $\|\varphi\|_{+}$ or $\|\varphi\|_{-}$when $|\mathfrak{R} z|$ is large enough. Thus for any $r, 0<r<a$, there exist constants $M_{1}$ and $M_{2}$ such that

$$
M_{1}\|\varphi\|_{+} \leqq\|\varphi\|_{z} \leqq M_{2}\|\varphi\|_{+}, \quad\left|\mathfrak{I}_{z}\right| \leqq r .
$$

The operator $H(z) \in \mathscr{L}\left(X_{+}, \mathscr{H}\right)$ has limits when $\left|\mathfrak{R}_{z}\right|$ diverges. For any $r, 0<r<a$, there exists a constant $M_{+}$such that

$$
\|H(z)\|_{+} \leqq M_{+}, \quad|\mathfrak{J} z| \leqq r+\frac{(a-r)}{2} .
$$

Using Cauchy formula and (2.13) we have

$$
\left\|H^{\prime}(t+i s) \varphi\right\| \leqq M_{+}^{\prime}\|\varphi\|_{+}, \quad|s| \leqq r .
$$

On the other hand, if $|t|$ is large enough, we can use condition II instead of (2.13), and apply Cauchy formula to the applications $\left(H(z)-H^{+}\right) \varphi \operatorname{or}\left(H(z)-H^{-}\right) \varphi$ as above. We get for $|t|$ large enough

$$
\left\|H^{\prime}(t+i s) \varphi\right\| \leqq \frac{C^{\prime}}{(1+|t|)^{1+\alpha}}\|\varphi\|_{+}, \quad|s| \leqq r
$$


and

$$
\left\|H^{\prime}(t+i s) \varphi\right\| \leqq \frac{C^{\prime}}{(1+|t|)^{1+\alpha}}\|\varphi\|_{-}, \quad|s| \leqq r .
$$

In (2.14), (2.15) and (2.16) the constants depend on $r$ only. Since we can compare any norm with $\|\varphi\|_{+}$by (2.12), we have the following lemma.

Lemma 2.1. If $H(z)$ satisfies conditions $I$ and $I I$ and if $0<r<a$, then there exists a positive integrable function $c(t)$ (which behaves like $|t|^{-(1+x)}$ at infinity) such that for all $t \in \mathbb{R}$ and $|s| \leqq r$ and all $z^{\prime}$,

$$
\left\|H^{\prime}(t+i s) \varphi\right\| \leqq c(t)\|\varphi\|_{z^{\prime}}
$$

From Lemma (2.1) and for $|s| \leqq r$ we immediately have the estimate

$$
\|(H(t+i s)-H(t)) \varphi\| \leqq|s| c(t)\|\varphi\|_{t}
$$

with $c(t)$ an integrable function. Let $\lambda \in \rho(t)$. The operator $H(t) R(t, \lambda)$ is a bounded operator and (2.17) implies

$$
\begin{aligned}
\|(H(t+i s)-H(t)) R(t, \lambda)\| & \leqq|s| c(t)(\|R(t, \lambda)\|+\|H(t) R(t, \lambda)\|) \\
& \equiv|s| c(t) d(t, \lambda) .
\end{aligned}
$$

When $|s| c(t) d(t, \lambda)<1, \lambda$ belongs also to the resolvent set $\rho(t+i s)$ and we have

$$
\|R(t+i s, \lambda)-R(t, \lambda)\| \leqq\|R(t, \lambda)\| \frac{|s| c(t) d(t, \lambda)}{1-|s| c(t) d(t, \lambda)}
$$

In particular if $\lambda \in \rho\left(H^{+}\right)$then $\lambda \in \rho(t+i s)$ for $t$ large enough and for any $\beta<1+\alpha$,

$$
\lim _{t \rightarrow \infty}|t|^{\beta} \sup _{|s|<a}\|R(t+i s, \lambda)-R(+, \lambda)\|=0 .
$$

By choosing the width of the band small enough the spectrum $\sigma(z)$ is separated into two parts $\sigma_{1}(z)$ and $\sigma_{2}(z)$ and we can find a path $\Gamma$ encircling the bounded set $\sigma_{1}(z)$ so that the spectral projector $P_{1}(z)$ can be written

$$
P_{1}(z)=-\frac{1}{2 \pi i} \oint_{\Gamma} R(z, \lambda) d \lambda .
$$

From (2.21) and (2.20) we get Lemma (2.2).

Lemma 2.2. If the width of $S_{a}$ is small enough then the projectors $P_{1}(z)$ and $P_{2}(z)=1-P_{1}(z)$ are holomorphic on $S_{a}$ and have limits $P_{k}( \pm), k=1,2$ when $|\mathfrak{R} z| \rightarrow \infty$. Moreover, for any $\beta<1+\alpha$,

$$
\lim _{t \rightarrow \infty}|t|^{\beta} \sup _{|s|<a}\left\|P_{k}(t+i s)-P_{k}(+)\right\|=0
$$

and for any $r, 0<r<a$ and any integer $n$

$$
\lim _{t \rightarrow \infty}|t|^{\beta} \sup _{|s| \leqq r}\left\|P_{k}^{(n)}(t+i s)\right\|=0 .
$$

Similar statements hold for $t \rightarrow-\infty$. 
Lemma 2.3. Let $0<r<a$. Let $\lambda \in \rho(z)$ for all $z$ in the band, with $|\Im z| \leqq r$ (e.g. $\lambda$ is negative and $|\lambda|$ large enough). Then for all $z$ with $|\Im z| \leqq r H^{\prime}(z) R(z, \lambda)$ is a bounded holomorphic operator. Moreover, there exists a constant $N$ such that

$$
\left\|H^{\prime}(z) R(z, \lambda)\right\| \leqq N c(t), \quad|\mathfrak{I} z| \leqq r .
$$

Proof. We decompose the operator as

$$
H^{\prime}(z) R(z, \lambda)=H^{\prime}(z) R(0, \lambda)(H(0)-\lambda) R(z, \lambda) .
$$

The factor $H^{\prime}(z) R(0, \lambda)$ is a bounded holomorphic operator by condition $I$ and Lemma (2.1). The other factor $(H(0)-\lambda) R(z, \lambda)$ is a bounded operator, locally uniformly bounded in $z$. Since $(H(0)-\lambda) R(z, \lambda)$ is the inverse of the operator $(H(z)-\lambda) R(0, \lambda)$ which is a holomorphic bounded operator, $(H(0)-\lambda) R(z, \lambda)$ is itself a holomorphic bounded operator. From Lemma (2.1) we have

$$
\begin{aligned}
\left\|H^{\prime}(z) R(z, \lambda) \varphi\right\| & \leqq c(t)\|R(z, \lambda) \varphi\|_{z} \\
& \leqq c(t)(\|R(z, \lambda)\|+1+|\lambda|\|R(z, \lambda)\|)\|\varphi\| .
\end{aligned}
$$

The lemma follows therefore from (2.23) and (2.19).

\section{Adiabatic Evolution}

In this section we follow mainly [7], Chaps. II and IV. Let $\varphi(t)$ be the solution of the Schrödinger equation

$$
i \varepsilon \frac{\partial}{\partial t} \varphi(t)=H(t) \varphi(t), \quad \varphi\left(t^{\prime}\right)=\varphi^{*} \in D
$$

Our conditions on $H$ imply the existence of a unitary operator $U\left(t, t^{\prime}\right)$ defined for all real $t$ and $t^{\prime}$, strongly continuous in $t$ and $t^{\prime}$, which leaves the domain $D$ invariant. For all $t_{1}, t_{2}, t_{3}$ we have

$$
U\left(t_{1}, t_{2}\right) U\left(t_{2}, t_{3}\right)=U\left(t_{1}, t_{3}\right), \quad U\left(t_{1}, t_{1}\right)=1 .
$$

On $D, U$ is strongly differentiable in $t$ and $t^{\prime}$,

$$
i \varepsilon \frac{\partial}{\partial t} U\left(t, t^{\prime}\right)=H(t) U\left(t, t^{\prime}\right)
$$

and

$$
i \varepsilon \frac{\partial}{\partial t^{\prime}} U\left(t, t^{\prime}\right)=-U\left(t, t^{\prime}\right) H\left(t^{\prime}\right)
$$

The solution of (3.1) is given by $\varphi(t)=U\left(t, t^{\prime}\right) \varphi^{*}$. The second evolution is the adiabatic evolution. It is the evolution $V\left(t, t^{\prime}\right)$ related to the equation

$$
i \varepsilon \frac{\partial}{\partial t} \psi(t)=\left(H(t)+i \varepsilon\left[P_{1}^{\prime}(t), P_{1}(t)\right]\right) \psi(t), \quad \psi\left(t^{\prime}\right)=\psi^{*}
$$

This evolution has the same general properties as the evolution $U\left(t, t^{\prime}\right)$. 
Lemma 3.1. The evolution $V\left(t, t^{\prime}\right)$ is compatible with the decomposition of the Hilbert space $\mathscr{H}$ into $\mathscr{H}_{1}(t)$ and $\mathscr{H}_{2}(t)$ :

$$
P_{k}(t) V\left(t, t^{\prime}\right)=V\left(t, t^{\prime}\right) P_{k}\left(t^{\prime}\right), \quad k=1,2 .
$$

The proof is given in [7], Chap. 4. The two evolutions $U\left(t, t^{\prime}\right)$ and $V\left(t, t^{\prime}\right)$ depend on $\varepsilon$. However we do not write this dependence explicitly. The next result describes the adiabatic limit $\varepsilon \rightarrow 0$ for Eq. (3.1).

Theorem 3.1. Under the conditions $I, I I$ and $I I I$, there exists a constant $M$ such that for all $t^{\prime} \in \mathbb{R}$,

$$
\sup _{t \in \mathbb{R}}\left\|U\left(t, t^{\prime}\right)-V\left(t, t^{\prime}\right)\right\| \leqq M \varepsilon
$$

Remark. This theorem is valid under weaker hypothesis (see Sect. (1)). From it we have immediately that $\mathscr{P}_{21}\left(t, t^{\prime}\right)=O\left(\varepsilon^{2}\right)$ uniformly in $t$ and $t^{\prime}$. Theorem (3.1) is not new (see e.g. $[7,6]$ ). Some ideas of the proof are used later on.

Sketch of the Proof. Let $t^{\prime}=0$ and $U(t)=U(t, 0), V(t)=V(t, 0)$. For any $\varphi \in D$ we define

$$
x(t)=V(t)^{-1} U(t) \varphi .
$$

The function $x(t)$ satisfies the equation

$$
\begin{aligned}
x^{\prime}(t) & =-V(t)^{-1}\left[P_{1}^{\prime}(t), P_{1}(t)\right] V(t) x(t) \\
& \equiv K(t) x(t) .
\end{aligned}
$$

The operator $K(t)$ is a bounded, anti-self-adjoint operator. It is strongly continuous in $t$ and $\|K(t)\|$ is integrable on the real axis (Lemma (2.2)) uniformly in $\varepsilon$ since for $t \in \mathbb{R} V(t)$ is unitary. Equation (3.7) is equivalent to the Volterra equation

$$
x(t)=x\left(t_{0}\right)+\int_{t_{0}}^{t} K(u) x(u) d u .
$$

From Lemma (3.1) we have

$$
P_{1}(0) K(s)=K(s) P_{2}(0)
$$

and

$$
P_{2}(0) K(s)=K(s) P_{1}(0) .
$$

Using (3.9) and (3.10) we can express Eq. (3.8) as a system of two equations. Let $x_{k}(t)=P_{k}(0) x(t), k=1,2$. Then

$$
x_{1}(t)=x_{1}\left(t_{0}\right)+\int_{t_{0}}^{t} K_{12}(u) x_{2}(u) d u
$$

and

$$
x_{2}(t)=x_{2}\left(t_{0}\right)+\int_{t_{0}}^{t} K_{21}(u) x_{1}(u) d u,
$$

where we have (by Lemma (3.1))

$$
K_{12}(s)=P_{1}(0) V^{-1}(s) P_{1}^{\prime}(s) V(s) P_{2}(0)
$$


and

$$
K_{21}(s)=-P_{2}(0) V^{-1}(s) P_{1}^{\prime}(s) V(s) P_{1}(0) .
$$

The next step is to perform an integration by parts in both (3.11) and (3.12). From the result we get immediately

$$
\begin{aligned}
\|(U(t, 0)-V(t, 0)) \varphi\| & =\|V(t, 0) x(t)-V(t, 0) \varphi\| \\
& \leqq\|\varphi\| \varepsilon M . \square
\end{aligned}
$$

Lemma 3.2 (Integration by parts formula). Let $B(t)$ be a bounded operator, strongly $C^{1}$ and let $x(t) \in D \forall t$ and be $C^{1}$. We define

$$
\widetilde{B(t)}=\frac{1}{2 \pi i} \oint_{\Gamma} R(t, \lambda) B(t) R(t, \lambda) d \lambda
$$

where $\Gamma$ is a path surrounding the bounded part of the spectrum $\sigma_{1}(t)$. Then

1. $\widetilde{B(t)}$ is strongly $C^{1}$ and maps $\mathscr{H}$ into D. Moreover

$$
P_{k}(t) \widetilde{B(t)} P_{k}(t)=0, \quad k=1,2
$$

2.

$$
\begin{aligned}
& \int_{t^{\prime}}^{t} P_{1}(0) V(s)^{-1} B(s) V(s) P_{2}(0) x(s) d s=-\left.i \varepsilon P_{1}(0) V(s)^{-1} \widetilde{B(s)} V(s) P_{2}(0) x(s)\right|_{t^{\prime}} ^{t} \\
& +i \varepsilon \int_{I^{\prime}}^{t} P_{1}(0) V(s)^{-1}\left(\frac{d}{d s} \widetilde{B(s)}\right) V(s) P_{2}(0) x(s) d s \\
& +i \varepsilon \int_{t^{\prime}}^{t} P_{1}(0) V(s)^{-1} \widetilde{B(s)} V(s) P_{2}(0) \frac{d}{d s} x(s) d s .
\end{aligned}
$$

3. An analogous formula holds for $\int_{t^{\prime}}^{t} P_{2}(0) V(s)^{-1} B(s) V(s) P_{1}(0) x(s) d s$. It is obtained by exchanging $P_{1}(0)$ and $P_{2}(0)$ and changing the sign on the right-hand side in the above formula.

Remark. This lemma is proved in [6] except part 1) which simplifies the formula.

\section{Bounded Operators}

We prove in this section our result for bounded operators. Let us suppose that $H(z)$ is a bounded operator which satisfies conditions I, II, and III and that all conclusions of Lemmas (2.1) to (2.3) are valid. As in Sect. (3) we choose $t^{\prime}=0$ and put $U(t)=U(t, 0) V(t)=V(t, 0)$. Let

$$
A(t)=V^{-1}(t) U(t)
$$

This operator satisfies the equation

$$
A^{\prime}(t)=K(t) A(t), \quad A(0)=\mathbb{1} .
$$


We now take complex time, i.e. we take $t=z \in S_{a}$ and we consider Eq. (4.2) on $S_{a}$

$$
A^{\prime}(z)=\frac{d}{d z} A(z)=K(z) A(z), \quad A(0)=\mathbb{1} .
$$

The operator $K(z)$ is holomorphic on $S_{a}$ because the operator $V(z)$ has an analytic extension on $S_{a}$. Indeed, we can define for $z \in S_{a}$,

$$
V(z)=\mathbb{1}+\int_{0}^{z}\left(-i \varepsilon^{-1} H\left(z^{\prime}\right)+\left[P_{1}^{\prime}\left(z^{\prime}\right), P_{1}\left(z^{\prime}\right)\right]\right) V\left(z^{\prime}\right) d z^{\prime} .
$$

The path of integration in (4.4) is any path in $S_{a}$ from 0 to $z$. By choosing suitably the path of integration we obtain immediately from (4.4),

$$
\|V(z)\| \leqq \text { Const } \exp \left(\frac{r \sup _{z:|\mathfrak{J} z| \leqq r}\|H(z)\|}{\varepsilon}\right), \quad|\mathfrak{I} z| \leqq r,
$$

where $0<r<a$. From (4.5) and Lemma (2.2) it follows that $\|K(z)\|$ is integrable

$$
\sup _{|s| \leqq r} \int_{-\infty}^{+\infty}\|K(t+i s)\| d t<\infty .
$$

Equation (4.3) is equivalent to a Volterra equation and can be solved iteratively. Thus its solution $A(z)$ is holomorphic in $S_{a}$ and by the integrability condition (4.6) $A(z)$ has limits $A(+)$ and $A(-)$ when $|\mathfrak{R} z| \rightarrow \infty$,

$$
\lim _{t \rightarrow \pm \infty} \sup _{|s| \leqq r}\|A(t+i s)-A( \pm)\|=0 .
$$

The operators $A(+)$ and $A(-)$ are unitary since on the real axis $K(t)$ is anti selfadjoint.

Lemma 4.1. Let $\varphi(t)$ be a solution of the Schrödinger equation with $\varphi(0)=\varphi^{*} \in D$ and such that

$$
\lim _{t \rightarrow-\infty}\left\|P_{2}(t) \varphi(t)\right\|=0 .
$$

Then there exists a unique $\psi^{*} \in \mathscr{H}_{1}(0)$ such that $\varphi^{*}=A(-)^{-1} \psi^{*}$.

Proof. We can write the solution $\varphi(t)$ as

$$
\varphi(t)=V(t) A(t) \varphi^{*}
$$

By Lemma (3.1)

$$
\left\|P_{2}(t) V(t) A(t) \varphi^{*}\right\|=\left\|V(t) P_{2}(0) A(t) \varphi^{*}\right\|=\left\|P_{2}(0) A(t) \varphi^{*}\right\| .
$$

By hypothesis we have

$$
\lim _{t \rightarrow-\infty}\left\|P_{2}(0) A(t) \varphi^{*}\right\|=\left\|P_{2}(0) A(-) \varphi^{*}\right\|=0
$$

and therefore $\psi^{*}=A(-) \varphi^{*} \in \mathscr{H}_{1}(0)$.

We can give a convenient expression for the adiabatic invariant $\mathscr{P}_{21}(\infty,-\infty)$. By Lemma (4.1) any solution $\varphi(t)$ of the Schrödinger equation, which is normalized 
and satisfies the boundary condition

$$
\lim _{t \rightarrow-\infty}\left\|P_{2}(t) \varphi(t)\right\|=0
$$

can be written

$$
\varphi(t)=U(t) A(-)^{-1} \psi^{*}, \quad\left\|\psi^{*}\right\|=1 .
$$

By a computation similar to that of the proof of Lemma (4.1) we get

$$
\lim _{t \rightarrow \infty}\left\|P_{2}(t) \varphi(t)\right\|=\left\|P_{2}(0) A(+) A(-)^{-1} P_{1}(0) \psi^{*}\right\| .
$$

Therefore we have

$$
\mathscr{P}_{21}(\infty,-\infty)=\left\|P_{2}(0) A(+) A(-)^{-1} P_{1}(0)\right\|^{2} .
$$

Remark. The solution $A(t)$ of (4.2) is normalized at $t=0$. Since we have the integrability condition (4.6) we can work with a solution $A(t,-\infty)$ normalized at $t=-\infty$. By definition $A(t,-\infty)$ is a solution of $(4.2)$ with $\lim _{t \rightarrow-\infty} A(t,-\infty)=\mathbb{1}$, and we can express (4.14) as

$$
\mathscr{P}_{21}(\infty,-\infty)=\left\|P_{2}(0) A(\infty,-\infty) P_{1}(0)\right\|^{2} .
$$

Notice that this formula is also true in the unbounded case, since we are on the real axis.

The next lemma is the generalization of Lemma (3.1) in the complex plane. The proof is the same.

Lemma 4.2. For all $z \in S_{a}$ we have

$$
P_{k}(z) V(z)=V(z) P_{k}(0), \quad k=1,2 .
$$

Proof of Theorem 1.1. We must estimate the norm of the operator $P_{2}(0) A(\infty,-\infty) P_{1}(0)$. The operator $A(t,-\infty)$ is solution of the equation

$$
A(t,-\infty)=\mathbb{1}+\int_{-\infty}^{t} K(u) A(u,-\infty) d u .
$$

This operator has an analytic extension on $S_{a}$ since $A(t,-\infty)=A(t) A(-)^{-1}$. Moreover, by (4.7) we know that

$$
\lim _{\tau \rightarrow \infty} A(\gamma(\tau),-\infty)=A(\infty,-\infty)
$$

if $\gamma(\tau)=\gamma_{1}(\tau)+i \gamma_{2}(\tau)$ is a smooth path in $S_{a}$, parametrized by $\tau$, such that

$$
\gamma_{1}(\tau)=\tau, \quad \gamma_{2}(\tau)>0, \quad \lim _{\tau \rightarrow \pm \infty} \gamma_{2}(\tau) \geqq \kappa>0 .
$$

Therefore we consider $A(\gamma(\tau),-\infty)$ as a function of $\tau$. This is the solution of the equation

$$
A(\gamma(\tau),-\infty)=\mathbb{1}+\int_{-\infty}^{t} K(\gamma(u)) A(\gamma(u),-\infty) \dot{\gamma}(u) d u,
$$

where $\dot{\gamma}(\tau)=\frac{d}{d \tau}\left(\gamma_{1}(\tau)+i \gamma_{2}(\tau)\right)$. Let $x(z)$ be the solution of $(4.3)$ with initial condition 
$x(0)=A(-)^{-1} x^{*}, x^{*} \in \mathscr{H}_{1}(0)$ and $\left\|x^{*}\right\|=1$. Then we have

$$
x(\gamma(\tau))=A(\gamma(\tau),-\infty) x^{*},
$$

and we must estimate (see (4.15)) $\left\|x_{2}(\gamma(\tau))\right\|$ as $\tau \rightarrow \infty$.

In order to do that we introduce new quantities $\theta_{k}(z)$. Let $\lambda(z)$ be the function defined by

$$
\lambda(t+i s)=i s e_{1}(t),
$$

where $e_{1}(t)$ is the function of condition III. We define

$$
\theta_{k}(z)=\exp \left(i \varepsilon^{-1} \lambda(z)\right) V(z) x_{k}(z), \quad k=1,2 .
$$

Lemma 4.3. There exist a path $\gamma$ satisfying conditions (4.18), in particular $\lim _{\tau \rightarrow \infty} \gamma_{2}(\tau)=\kappa>0$, and a constant $M$ such that

$$
\left\|\theta_{1}(\gamma(\tau))\right\| \leqq M, \quad\left\|\theta_{2}(\gamma(\tau))\right\| \leqq \varepsilon M, \quad \tau \in \mathbb{R}
$$

for $\varepsilon$ small enough.

We first finish the proof of the theorem and then prove Lemma (4.3). Let $V\left(z, z^{\prime}\right)$ be the solution of the equation

$$
\frac{d}{d z} V\left(z, z^{\prime}\right)=\left(-i \varepsilon^{-1} H(z)+\left[P_{1}^{\prime}(z), P_{1}(z)\right]\right) V\left(z, z^{\prime}\right)
$$

with initial condition $V\left(z^{\prime}, z^{\prime}\right)=\mathbb{1}$. For $z=\tau+i \gamma_{2}(\tau)$ we have

$$
V(z)^{-1}=V\left(\tau+i \gamma_{2}(\tau), 0\right)^{-1}=V(\tau, 0)^{-1} V\left(\tau+i \gamma_{2}(\tau), \tau\right)^{-1}
$$

The operator $V(\tau+i u, \tau)^{-1}$ is solution of the equation

$$
\frac{d}{d u} V(\tau+i u, \tau)^{-1}=-V(\tau+i u, \tau)^{-1}\left(\varepsilon^{-1} H(\tau+i u)+i\left[P_{1}^{\prime}(\tau+i u), P_{1}(\tau+i u)\right]\right)
$$

on the interval $\left[0, \gamma_{2}(\tau)\right]$, with initial condition at $u=0 V(\tau, \tau)=\mathbb{1}$. By Lemmas (4.2) and (4.3), formula (4.24) and $\left\|V(\tau, 0)^{-1}\right\|=1$,

$$
\begin{aligned}
\left\|x_{2}(\gamma(\tau))\right\| & =\exp \left(\gamma_{2}(\tau) \varepsilon^{-1} e_{1}(\tau)\right)\left\|V(\gamma(\tau))^{-1} \theta_{2}(\gamma(\tau))\right\| \\
& =\exp \left(\gamma_{2}(\tau) \varepsilon^{-1} e_{1}(\tau)\right)\left\|V(\gamma(\tau))^{-1} P_{2}(\gamma(\tau)) \theta_{2}(\gamma(\tau))\right\| \\
& \leqq \varepsilon M \exp \left(\gamma_{2}(\tau) \varepsilon^{-1} e_{1}(\tau)\right)\left\|V(\gamma(\tau), \tau)^{-1} P_{2}(\gamma(\tau))\right\| .
\end{aligned}
$$

Using the differential equation (4.25), and Lemma (2.2) we get from (4.26),

$$
\begin{aligned}
\lim _{\tau \rightarrow \infty}\left\|x_{2}(\gamma(\tau))\right\| & \leqq \varepsilon M \exp \left(\gamma_{2}(\infty) \varepsilon^{-1} e_{1}(\infty)\right)\left\|\exp \left(-\gamma_{2}(\infty) \varepsilon^{-1} H(+) P_{2}(+)\right)\right\| \\
& \leqq \varepsilon M \exp \left(-\varepsilon^{-1} \kappa\left(e_{2}(\infty)-e_{1}(\infty)\right)\right)
\end{aligned}
$$

since we have with $H_{2}(\tau)=H(\tau) P_{2}(\tau)$ for any $\tau \in \mathbb{R}$,

$$
\left\|\exp \left(-\gamma_{2}(\tau) \varepsilon^{-1} H_{2}(\tau)\right)\right\| \leqq \exp \left(-\gamma_{2}(\tau) \varepsilon^{-1} \inf _{\|\varphi\|=1, \varphi \in \mathscr{H}_{2}(\tau)}\left\langle\varphi \mid H_{2}(\tau) \varphi\right\rangle\right) .
$$

Proof of Lemma 4.3. Let us consider the quantities $x_{k}(\gamma(\tau))$ along a path $\gamma$. They 
satisfy the equation (see (3.11) to (3.14))

$$
x_{1}(\gamma(\tau))=x^{*}+\int_{-\infty}^{\tau} K_{12}(\gamma(u)) x_{2}(\gamma(u)) \dot{\gamma}(u) d u
$$

and

$$
x_{2}(\gamma(\tau))=\int_{-\infty}^{\tau} K_{21}(\gamma(u)) x_{1}(\gamma(u)) \dot{\gamma}(u) d u
$$

since $x^{*} \in \mathscr{H}_{1}(0)$. We perform an integration by parts in (4.30) (see Lemma (3.2)) and we write $x_{k}(u)$ for $x_{k}(\gamma(u))$ etc. We get

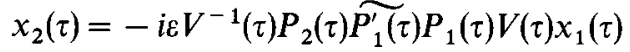

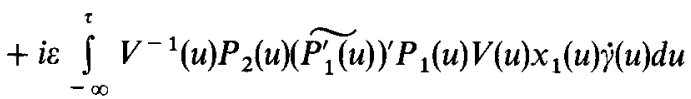

$$
\begin{aligned}
& +i \varepsilon \int_{-\infty}^{\tau} V^{-1}(u) P_{2}(u) \widetilde{P_{1}^{\prime}(u)} P_{1}(u) P_{1}^{\prime}(u) P_{2}(u) V(u) x_{2}(u) \dot{\gamma}(u) d u
\end{aligned}
$$

(In (4.31)' denotes the derivative with respect to the complex variable $z$.) We write the result using $\theta_{k}$ of (4.22) and $Q$ defined by

$$
Q(z)=\exp \left(i \varepsilon^{-1} \lambda(z)\right) V(z), \quad z \in S_{a} .
$$

We get

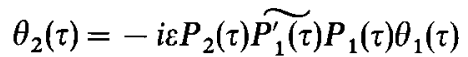

$$
\begin{aligned}
& +i \varepsilon \int_{-\infty}^{\tau} Q(\tau) Q^{-1}(u) P_{2}(u)\left(\widetilde{P_{1}^{\prime}(u)}\right)^{\prime} P_{1}(u) \theta_{1}(u) \dot{\gamma}(u) d u \\
& +i \varepsilon \int_{-\infty}^{\tau} Q(\tau) Q^{-1}(u) P_{2}(u) \widetilde{P_{1}^{\prime}(u)} P_{1}(u) P_{1}^{\prime}(u) P_{2}(u) \theta_{2}(u) \dot{\gamma}(u) d u
\end{aligned}
$$

Equation (4.29) becomes

$$
\theta_{1}(\tau)=Q(\tau) x^{*}+\int_{-\infty}^{\tau} Q(\tau) Q^{-1}(u) P_{1}(u) P_{1}^{\prime}(u) P_{2}(u) \theta_{2}(u) \dot{\gamma}(u) d u .
$$

On the path $\gamma$ the operator $Q(t)=Q(\gamma(\tau))$ satisfies the equation

$$
\frac{d}{d \tau} Q(\tau)=D(\tau) Q(\tau)
$$

with

$$
D(\tau)=-i \varepsilon^{-1} H(\gamma(\tau)) \dot{\gamma}(\tau)+i \varepsilon^{-1} \frac{d}{d \tau} \lambda(\gamma(\tau)) \mathbb{1}+\left[P_{1}^{\prime}(\gamma(\tau)), P_{1}(\gamma(\tau))\right] \dot{\gamma}(\tau) .
$$

The operator $Q(\tau) Q(u)^{-1} P_{2}(u)$ is a solution of (4.35) with initial condition at $\tau=u$ given by $P_{2}(u)$. By Lemma (4.2) we have

$$
Q(\tau) Q(u)^{-1} P_{2}(u)=P_{2}(\tau) Q(\tau) Q(u)^{-1} P_{2}(u) .
$$


Therefore $Q(\tau) Q(u)^{-1} P_{2}(u)$ is a solution of the simpler equation

$$
\frac{d}{d \tau}\left(Q(\tau) Q(u)^{-1} P_{2}(u)\right)=D_{2}(\tau)\left(Q(\tau) Q(u)^{-1} P_{2}(u)\right)
$$

with

$$
D_{2}(\tau) \equiv D_{2}(\gamma(\tau))=D(\gamma(\tau)) P_{2}(\gamma(\tau)) .
$$

Similarly $Q(\tau) Q(u)^{-1} P_{1}(u)$ is solution of the simpler equation

$$
\frac{d}{d \tau}\left(Q(\tau) Q(u)^{-1} P_{1}(u)\right)=D_{1}(\tau)\left(Q(\tau) Q(u)^{-1} P_{1}(u)\right)
$$

with

$$
D_{1}(\tau) \equiv D_{1}(\gamma(\tau))=D(\gamma(\tau)) P_{1}(\gamma(\tau)) .
$$

The main problem is to control the norms of the operators $Q(\tau) Q(u)^{-1} P_{k}(u)$, $k=1,2$. We say that a path $\gamma$ is dissipative for Eq. (4.33) if there exists a constant $C_{0}$ independent of $\varepsilon$ such that

$$
\left\|Q(\tau) Q(u)^{-1} P_{2}(u)\right\| \leqq C_{0}, \quad \forall \tau \geqq u .
$$

Similarly, $\gamma$ is dissipative for Eq. (4.34) if along $\gamma$

$$
\left\|Q(\tau) Q(u)^{-1} P_{1}(u)\right\| \leqq C_{0}, \quad \forall \tau \geqq u .
$$

One natural way to find a path $\gamma$ which is dissipative for (4.33) would be to require that

$$
\mathfrak{R}\left\langle\varphi \mid D_{2}(\tau) \varphi\right\rangle \leqq 0, \quad \forall \varphi \in \mathscr{H},
$$

since this condition would imply (4.42) with $C_{0}=1$. However such a condition cannot be verified for all $\varphi \in \mathscr{H}$, but we show in Sect. (5) that a related condition to (4.44) implies (4.42) with $C_{0} \neq 1$ usually. Moreover there is another difficulty. We cannot find a single path which is dissipative for both Eqs. (4.33) and (4.34). We proceed as follows. In Sect. (5) we show that there is a path $\gamma$ which is dissipative for (4.33) and which satisfies the conditions (4.18), provided the width of the band is small enough (Lemma (5.6)). Let $\Delta$ be the region of the complex plane between the real axis and $\gamma$ :

$$
\Delta=\left\{t+i s ; t \in \mathbb{R}, 0 \leqq s \leqq \gamma_{2}(t)\right\} .
$$

Let $z=z_{1}+i z_{2}$ be a point in $\Delta$. We introduce two paths $\hat{\gamma}$ and $\tilde{\gamma}$ (see Fig. (1))

$$
\hat{\gamma}(\tau)= \begin{cases}\tau, & -\infty<\tau \leqq z_{1} \\ z_{1}+i\left(\tau-z_{1}\right), & z_{1}<\tau \leqq z_{1}+z_{2}\end{cases}
$$

and

$$
\tilde{\gamma}(\tau)=\left\{\begin{array}{ll}
\gamma(\tau), & -\infty<\tau \leqq z_{1} \\
\gamma\left(z_{1}\right)-i\left(\tau-z_{1}\right), & z_{1}<\tau \leqq z_{1}+\left(\gamma_{2}\left(z_{1}\right)-z_{2}\right)
\end{array} .\right.
$$

We prove (Lemma (5.5)) that $\hat{\gamma}$ is dissipative for Eq. (4.34) and $\tilde{\gamma}$ is dissipative for Eq. (4.33). The fact that $\hat{\gamma}$ is dissipative for $\tau \leqq z_{1}$ is trivial since for these values of $\tau$ the operator $D$ is anti self-adjoint. 


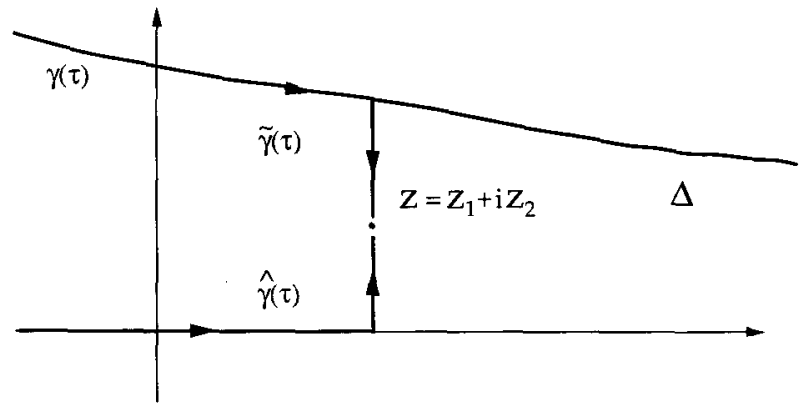

Fig. 1. The paths $\hat{\gamma}$ and $\tilde{\gamma}$ in $\Delta$

We can now finish the proof. Let

$$
\left\|\mid \theta_{k}\right\|\left\|=\sup _{z \in \Delta}\right\| \theta_{k}(z) \|, \quad k=1,2 .
$$

Using the fact that $\tilde{\gamma}$ is dissipative we have for $\tau=z_{1}+z_{2}$,

$$
\left\|\theta_{2}(z)\right\| \leqq \varepsilon M^{\prime}\left(\left\|\theta_{1}\right\| \mid+\left\|\theta_{2}\right\| \|\right) .
$$

If we use the path $\hat{\gamma}$ in (4.34) we get for $\tau=z_{1}+z_{2}$,

$$
\left\|\theta_{1}(z)\right\| \leqq M^{\prime \prime}+M^{\prime}\left\|\theta_{2}\right\| \text {. }
$$

Indeed we can write

$$
\begin{aligned}
Q(\tau) x^{*} & =Q(\tau) Q\left(z_{1}\right)^{-1} Q\left(z_{1}\right) x^{*} \\
& =Q(\tau) Q\left(z_{1}\right)^{-1} P_{1}\left(z_{1}\right) Q\left(z_{1}\right) x^{*}
\end{aligned}
$$

since $x^{*} \in \mathscr{H}_{1}(0)$. But $\left\|Q\left(z_{1}\right)\right\|=1$. Thus $\left\|Q(\tau) x^{*}\right\| \leqq M^{\prime \prime}$, using the fact that $\left\|x^{*}\right\|=1$. The constants $M^{\prime}$ and $M^{\prime \prime}$ are independent of $\varepsilon$ and can be chosen independently of the paths $\tilde{\gamma}$ and $\hat{\gamma}$, i.e. they do not depend on $z \in \Delta$. Therefore we can take the supremum over $\Delta$ on the right-hand side of (4.49) and (4.50).

Remark. There is an analogous result for $\mathscr{P}_{12}(\infty,-\infty)$. In that case we perform an integration by parts in Eq. (4.34) and we use a dissipative path $\gamma$ for Eq. (3.34); $\gamma$ satisfies instead of (4.18):

$$
\gamma_{1}(\tau)=\tau, \quad \gamma_{2}(\tau)<0, \quad \lim _{\tau \rightarrow \pm \infty} \gamma_{2}(\tau) \leqq-\kappa<0
$$

\section{Dissipative Paths}

We come to the crucial point of the proof of Theorem (1.1), the existence of dissipative paths for Eqs. (4.33) and (4.34). We first establish a sufficient condition for a path $\gamma$ to be dissipative. 
Let $H(z)$ be a bounded operator satisfying conditions I, and II and III. Let $\tau \mapsto \gamma(\tau)=\gamma_{1}(\tau)+i \gamma_{2}(\tau)$ be some path inside $S_{a}$, parametrized by $\tau$. We consider Eq. (4.35)

$$
\frac{d}{d \tau} \phi(\tau)=D(\tau) \phi(\tau)
$$

with

$$
D(\tau)=-i \varepsilon^{-1} H(\gamma(\tau)) \dot{\gamma}(\tau)+i \varepsilon^{-1} \frac{d}{d \tau} \lambda(\gamma(\tau)) \mathbb{1}+\left[P_{1}^{\prime}(\gamma(\tau)), P_{1}(\gamma(\tau))\right] \dot{\gamma}(\tau) .
$$

We are interested in the solutions $\phi_{k}(\tau, u)$ of (5.1) with initial conditions at $u$, $\phi_{k}(u, u)=P_{k}(\gamma(u))$. With the notations of Sect. (4) they can be written as

$$
\phi_{k}(\tau, u)=Q(\tau) Q(u)^{-1} P_{k}(\gamma(u))
$$

An important property of these solutions is that

$$
P_{k}(\gamma(\tau)) \phi_{k}(\tau, u)=\phi_{k}(\tau, u) P_{k}(\gamma(u)) \text {. }
$$

We use this fact as follows ([7], Chap. IV). Let $W\left(z, z^{\prime}\right)$ be the solution of the equation

$$
\frac{d}{d z} W\left(z, z^{\prime}\right)=\left[P_{1}^{\prime}(z), P_{1}(z)\right] W\left(z, z^{\prime}\right)
$$

with initial condition $W\left(z^{\prime}, z^{\prime}\right)=\mathbb{1}$. By Lemma (2.2) $W\left(z, z^{\prime}\right)$ is holomorphic on $S_{a}$, uniformly bounded on $S_{a}$,

$$
\sup _{z, z^{\prime} \in S_{a}}\left\|W\left(z, z^{\prime}\right)\right\| \leqq M
$$

(provided the width of the band is small enough) and $W\left(z, z^{\prime}\right)^{-1}=W\left(z^{\prime}, z\right)$. We also have

$$
P_{k}(z) W\left(z, z^{\prime}\right)=W\left(z, z^{\prime}\right) P_{k}\left(z^{\prime}\right), \quad k=1,2 .
$$

Lemma 5.1. The operator $W\left(z, z^{\prime}\right)$ leaves the domain of the operator $H(z)$ invariant. Let $\hat{H}(z):=W(0, z) H(z) W(z, 0)$ be defined on $D$. Let $0<r<a$. Then there exists an integrable function $\hat{c}(t)$ such that

$$
\|(\hat{H}(t+i s)-\hat{H}(t)) \varphi\| \leqq|s| \hat{c}(t)(\|\hat{H}(t) \varphi\|+\|\varphi\|), \quad \varphi \in D,
$$

provided $|s| \leqq r$

Moreover, $\hat{H}(t) \varphi$ is holomorphic for each $\varphi \in D$.

The first part of the proof of Lemma (5.1) is essentially given in [7] p. 308.

Proof. We prove the lemma for $z^{\prime}=0$. By Lemmas (2.2) and (2.3) the operator

$$
G(z)=\left[P_{1}^{\prime}(z), P_{1}(z)\right]+P_{1}(z) H^{\prime}(z) R(z, \lambda) P_{1}(z)+P_{2}(z) H^{\prime}(z) R(z, \lambda) P_{2}(z)
$$

is a bounded holomorphic operator, provided that $\lambda$ is negative and $|\lambda|$ is large enough. Moreover, there exists a constant $N^{\prime}$ so that for $|s| \leqq r$,

$$
\|G(t+i s)\| \leqq N^{\prime} c(t)
$$


with $c(t)$ the integrable function of Lemma (2.1). Therefore we can define $S(z)$ by

$$
S^{\prime}(z)=G(z) S(z), \quad S(0)=\mathbb{1} .
$$

The solution $S(z)$ is holomorphic. Besides $S(z)$ we also introduce the operator

$$
F(z)=R(z, \lambda) S(z) \text {. }
$$

Let us compute the derivative of $F(z)$,

$$
F^{\prime}(z)=R^{\prime}(z, \lambda) S(z)+R(z, \lambda) G(z) S(z) .
$$

We know that

$$
P_{k}(z) R(z, \lambda)=R(z, \lambda) P_{k}(z), \quad k=1,2 .
$$

By differentiating this identity we get

$$
P_{k}^{\prime}(z) R(z, \lambda)+P_{k}(z) R^{\prime}(z, \lambda)=R^{\prime}(z, \lambda) P_{k}(z)+R(z, \lambda) P_{k}^{\prime}(z) .
$$

Now, using (5.14), (5.15) and $R^{\prime}(z, \lambda)=-R(z, \lambda) H^{\prime}(z) R(z, \lambda)$, we have

$$
\begin{aligned}
R(z, \lambda) P_{k}^{\prime}(z)+R(z, \lambda) P_{k}(z) H^{\prime}(z) R(z, \lambda) & =R(z, \lambda) P_{k}^{\prime}(z)-P_{k}(z) R^{\prime}(z, \lambda) \\
& =P_{k}^{\prime}(z) R(z, \lambda)-R^{\prime}(z, \lambda) P_{k}(z) .
\end{aligned}
$$

Hence we can write

$$
\begin{aligned}
R(z, \lambda) G(z) & =R(z, \lambda)\left(\sum_{k=1}^{2} P_{k}^{\prime}(z) P_{k}(z)+P_{k}(z) H^{\prime}(z) R(z, \lambda) P_{k}(z)\right) \\
& =\sum_{k=1}^{2} P_{k}^{\prime}(z) P_{k}(z) R(z, \lambda)-R^{\prime}(z, \lambda) .
\end{aligned}
$$

Therefore the operator $F(z)$ satisfies the differential equation

$$
F^{\prime}(z)=\left(\sum_{k=1}^{2} P_{k}^{\prime}(z) P_{k}(z)\right) F(z)=\left[P_{1}^{\prime}(z), P_{1}(z)\right] F(z) .
$$

At $z=0$ we have $F(0)=R(0, \lambda)$ and by the uniqueness of the solution of $(5.18)$ we have

$$
F(z)=W(z, 0) R(0, \lambda)=R(z, \lambda) S(z) .
$$

Therefore $W(z, 0)$ leaves the domain $D$ invariant.

By definition

$$
S(t+i s)-S(t)=i \int_{0}^{s} G(t+i u) S(t+i u) d u .
$$

Iterating this equality we have

$$
S(t+i s)-S(t)=\sum_{n \geqq 1}(i)^{n} \int_{0}^{s} d y_{1} \cdots \int_{0}^{y_{n-1}} d y_{n} G\left(t+i y_{1}\right) \cdots G\left(t+i y_{n}\right) S(t),
$$

and by (5.10)

$$
\|(S(t+i s)-S(t)) \varphi\| \leqq|s| N^{\prime} c(t) \exp \left(|s| N^{\prime} c(t)\right)\|S(t) \varphi\| .
$$


Using (5.19) we have

$$
\begin{aligned}
\|(\hat{H}(t+i s)-\hat{H}(t)) R(0, \lambda) \varphi\|= & \|(W(0, t+i s) S(t+i s)-W(0, t) S(t)) \varphi\| \\
\leq & \|W(0, t+i s)-W(0, t)\|\|S(t) \varphi\| \\
& +\|W(0, t+i s)\|\|(S(t+i s)-S(t)) \varphi\| .
\end{aligned}
$$

Since we can write

$$
W(0, t+i s)-W(0, t)=-i \int_{0}^{s} d y W(0, t+i y)\left[P_{1}^{\prime}(t+i y), P_{1}(t+i y)\right],
$$

we have by Lemma (2.2) and estimate (5.22) the existence of a constant $N^{\prime \prime}$ such that

$$
\begin{aligned}
\|(\hat{H}(t+i s)-\hat{H}(t)) R(0, \lambda) \varphi\| \leqq & |s| c(t) N^{\prime \prime}\|S(t) \varphi\| \\
= & |s| c(t) N^{\prime \prime}\|(H(t)-\lambda) R(t, \lambda) S(t) \varphi\| \\
= & |s| c(t) N^{\prime \prime}\|(H(t)-\lambda) W(t, 0) R(0, \lambda) \varphi\| \\
= & |s| c(t) N^{\prime \prime}\|W(t, 0)(\hat{H}(t)-\lambda) R(0, \lambda) \varphi\| \\
\leqq & |s| c(t) N^{\prime \prime}(\|\hat{H}(t) R(0, \lambda) \varphi\| \\
& +|\lambda|\|R(0, \lambda) \varphi\|) .
\end{aligned}
$$

Finally, if $\varphi \in D$,

$$
\hat{H}(z) \varphi=W(0, z) S(z) \psi+W(0, z) \lambda R(z, \lambda) S(z) \psi
$$

for a $\psi \in \mathscr{H}$, and this application is holomorphic because $W(0, z), S(z)$ and $R(z, \lambda)$ are bounded, holomorphic operators.

Let us introduce a new operator $Q_{0}(\tau)$ by putting

$$
Q_{0}(\tau)=W(0, \gamma(\tau)) Q(\tau)
$$

The solution $\phi_{k}(\tau, u)$ (see (5.3)) now reads

$$
\begin{aligned}
\phi_{k}(\tau, u) & =W(\gamma(\tau), 0) Q_{0}(\tau) Q_{0}(u)^{-1} W(0, \gamma(u)) P_{k}(\gamma(u)) \\
& =W(\gamma(\tau), 0) Q_{0}(\tau) Q_{0}(u)^{-1} P_{k}(0) W(0, \gamma(u)) .
\end{aligned}
$$

In order to prove that $\left\|\phi_{k}(\tau, u)\right\|$ is uniformly bounded for all $\tau \geqq u$ it is necessary and sufficient to prove that $\left\|Q_{0}(\tau) Q_{0}(u)^{-1} P_{k}(0)\right\|$ is uniformly bounded for all $\tau \geqq u$. The operator $Q_{0}(u)$ is solution of the equation

$$
\begin{aligned}
\frac{d}{d \tau} Q_{0}(\tau) & =-W(0, \gamma(\tau))\left[P_{1}^{\prime}(\tau), P_{1}(\tau)\right] \dot{\gamma}(\tau) Q(\tau)+W(0, \gamma(\tau)) D(\tau) Q(\tau) \\
& \equiv-i \varepsilon^{-1} \hat{T}(\tau) Q_{0}(\tau)
\end{aligned}
$$

where (see (5.2))

$$
\widehat{T}(\tau)=W(0, \gamma(\tau)) T(\tau) W(\gamma(\tau), 0)
$$

and

$$
\begin{aligned}
T(\tau) & =H(\gamma(\tau)) \dot{\gamma}(\tau)-\frac{d}{d \tau} \lambda(\gamma(\tau)) \mathbb{1} \\
& =H(\gamma(\tau))\left(\dot{\gamma}_{1}(\tau)+i \dot{\gamma}_{2}(\tau)\right)-i e_{1}^{\prime}\left(\gamma_{1}(\tau)\right) \gamma_{2}(\tau) \dot{\gamma}_{1}(\tau)-i e_{1}\left(\gamma_{1}(\tau)\right) \dot{\gamma}_{2}(\tau)
\end{aligned}
$$


Since we are interested only in the solutions $Q_{0}(\tau) Q_{0}(u)^{-1} P_{k}(0)$ of $(5.29)$ we can also consider them as solutions of the simpler equation

$$
\frac{d}{d \tau} Q_{0}(\tau)=-i \varepsilon^{-1} \hat{T}(\tau) P_{k}(0) Q_{0}(\tau)
$$

These solutions can therefore be considered as solutions of Eq. (5.32) in the fixed Hilbert space $\mathscr{H}_{k}(0)=P_{k}(0) \mathscr{H}$ with initial condition $\mathbb{1}_{\mathscr{H}_{k}(0)}$. It is therefore sufficient that $-i \varepsilon^{-1} \hat{T}(\tau) P_{k}(0)$, considered as an operator on $\mathscr{H}_{k}(0)$, be dissipative in order to have

$$
\left\|Q_{0}(\tau) Q_{0}(u)^{-1} P_{k}(0)\right\| \leqq 1, \quad \forall \tau \geqq u .
$$

We summarize these results in Lemma (5.2)

Lemma 5.2. Let $T(\tau)=H(\gamma(\tau)) \dot{\gamma}(\tau)-\frac{d}{d \tau} \lambda(\gamma(\tau)) \mathbb{1}$ and let $W(0, \gamma(\tau))$ be the operator defined by Eq. (5.5). A sufficient condition for a path $\gamma$ to be dissipative for Eqs. (4.33) and (4.34) is that for all $\varphi \in \mathscr{H}_{k}(0)$, all $\tau$

$$
\mathfrak{\Im}\langle\varphi \mid W(0, \gamma(\tau)) T(\tau) W(\gamma(\tau), 0) \varphi\rangle \leqq 0
$$

for $k=2$ resp. $k=1$.

We now apply this lemma to prove the existence of dissipative paths. We first consider a descending vertical path $\gamma$, i.e. a path of the type

$$
\tau \mapsto \gamma(\tau)=z^{*}-i \tau, \quad \tau \geqq 0, \quad z^{*}=z_{1}^{*}+i z_{2}^{*} \in S_{a} .
$$

Since here $\dot{\gamma}_{1}(\tau)=0$ and $\dot{\gamma}_{2}(\tau)=-1$, we must show (see (5.31)) in order that $\gamma$ be dissipative for (4.33) that

$$
\mathfrak{R}\left\langle\varphi \mid\left(\hat{H}(\gamma(\tau))-e_{1}\left(z_{1}^{*}\right)\right) \varphi\right\rangle \geqq 0, \quad \varphi \in \mathscr{H}_{2}(0) .
$$

This follows from Lemma (5.3).

Lemma 5.3. There exists a function $g_{2}(t)$, given by (5.42) such that for all $\varphi \in \mathscr{H}_{2}(0)$

$$
\mathfrak{R}\left\langle\varphi \mid\left(\hat{H}(t+i s)-e_{1}(t)\right) \varphi\right\rangle \geqq\left\langle\varphi \mid\left(\hat{H}(t)-e_{1}(t)\right) \varphi\right\rangle\left(1-|s| g_{2}(t)\right),
$$

and

$$
\mid \mathfrak{I}\left\langle\varphi|(\hat{H}(t+i s) \varphi\rangle| \leqq|s| g_{2}(t)\left\langle\varphi \mid\left(\hat{H}(t)-e_{1}(t)\right) \varphi\right\rangle,\right.
$$

provided that the width of the band $S_{a}$ is small enough.

Proof. We have for $\varphi \in \mathscr{H}_{2}(0)$,

$$
\begin{aligned}
\Re & \left\langle\varphi \mid\left(\hat{H}(t+i s)-e_{1}(t)\right) \varphi\right\rangle \\
& =\frac{1}{2}\left\langle\varphi \mid\left(\hat{H}(t+i s)+\hat{H}(t+i s)^{*}-2 e_{1}(t)\right) \varphi\right\rangle \\
& =\left\langle\varphi \mid\left(\hat{H}(t)-e_{1}(t)\right) \varphi\right\rangle+\frac{1}{2}\langle\varphi \mid((\hat{H}(t+i s)-\hat{H}(t))+(\hat{H}(t-i s)-\hat{H}(t))) \varphi\rangle
\end{aligned}
$$

since $W(z, 0)^{*}=W(0, \bar{z})$. By Lemma $(5.1)$,

$$
\begin{aligned}
\|(\hat{H}(t+i s)-\hat{H}(t)) \varphi\| & \leqq|s| \hat{c}(t)(\|\hat{H}(t) \varphi\|+\|\varphi\|) \\
& \leqq|s| \hat{c}(t)\left(\left\|\left(\hat{H}(t)-e_{1}(t)\right) \varphi\right\|+\left(1+\left|e_{1}(t)\right|\right)\|\varphi\|\right) .
\end{aligned}
$$


By condition III the operator $\left(\hat{H}(t)-e_{1}(t)\right)$ is positive on $\mathscr{H}_{2}(0)$. Thus

$$
\|(\hat{H}(t+i s)-\hat{H}(t)) \varphi\| \leqq \sqrt{2}|s| \hat{c}(t)\left\|\left(\hat{H}(t)-e_{1}(t)+1+\left|e_{1}(t)\right|\right) \varphi\right\| .
$$

At that point we use the following theorem (Theorem V.4.12. [8])

Theorem. Let $A_{1}$ be self-adjoint and non-negative. Let $A_{2}$ be symmetric with domain $D\left(A_{2}\right) \supset D\left(A_{1}\right)$ and $\left\|A_{2} \varphi\right\| \leqq\left\|A_{1} \varphi\right\|, \forall \varphi \in D\left(A_{1}\right)$. Then

$$
\left|\left\langle\varphi \mid A_{2} \varphi\right\rangle\right| \leqq\left\langle\varphi \mid A_{1} \varphi\right\rangle, \quad \varphi \in D\left(A_{1}\right) .
$$

We apply this theorem with $A_{2}=\frac{1}{2}(\hat{H}(t+i s)+\hat{H}(t-i s)-2 \hat{H}(t))$ and $A_{1}=\sqrt{2}|s| \hat{c}(t)\left(\hat{H}(t)-e_{1}(t)+1+\left|e_{1}(t)\right|\right)$, considered as operators on $\mathscr{H}_{2}(0)$. We get

$$
\begin{aligned}
& \mathfrak{R}\left\langle\varphi \mid\left(\hat{H}(t+i s)-e_{1}(t)\right) \varphi\right\rangle \\
& \quad \geqq\left\langle\varphi \mid\left(\hat{H}(t)-e_{1}(t)\right) \varphi\right\rangle(1-\sqrt{2}|s| \hat{c}(t))-\sqrt{2}|s| \hat{c}(t)\left(1+\left|e_{1}(t)\right|\right)\|\varphi\|^{2} .
\end{aligned}
$$

Since on $\mathscr{H}_{2}(0)$

$$
\hat{H}(t)-e_{1}(t) \geqq e_{2}(t)-e_{1}(t)
$$

we have

$$
\mathfrak{R}\left\langle\varphi \mid\left(\hat{H}(t+i s)-e_{1}(t)\right) \varphi\right\rangle \geqq\left\langle\varphi \mid\left(\hat{H}(t)-e_{1}(t)\right) \varphi\right\rangle\left(1-|s| g_{2}(t)\right)
$$

with

$$
g_{2}(t)=\sqrt{2} \hat{c}(t)\left(1+\frac{1+\left|e_{1}(t)\right|}{e_{2}(t)-e_{1}(t)}\right) .
$$

The second statement follows from the identity

$$
\mid \mathfrak{J}\left\langle\varphi|(\hat{H}(t+i s) \varphi\rangle|=\frac{1}{2}|\langle\varphi \mid i((\hat{H}(t+i s)-\hat{H}(t))-(\hat{H}(t-i s)-\hat{H}(t))) \varphi\rangle| .\right.
$$

Lemma 5.4. There exists a function $g_{1}(t)$, given by (5.46) so that for all $\varphi \in \mathscr{H}_{1}(0)$,

$$
\mathfrak{R}\left\langle\varphi \mid\left(e_{1}(t)-\hat{H}(t+i s)\right) \varphi\right\rangle \geqq\left\langle\varphi \mid\left(e_{1}(t)-\hat{H}(t)\right) \varphi\right\rangle\left(1-|s| g_{1}(t)\right)
$$

provided that the width of the band $S_{a}$ is small enough.

Proof. The proof is analogous to the proof of Lemma (5.3). The only difference is in the lower bound (5.40) which is now replaced by

$$
e_{1}(t)-\hat{H}(t) \geqq \delta_{1}^{*}
$$

if $e_{1}(t)-\hat{H}_{1}(t)$ is considered as an operator on $\mathscr{H}_{1}(0)$. The positive constant $\delta_{1}^{*}$ is given by

$$
\inf _{t} \operatorname{dist}\left(\sigma_{1}(t), e_{1}(t)\right)
$$

Therefore the function $g_{1}(t)$ is given by

$$
g_{1}(t)=\sqrt{2} \hat{c}(t)\left(1+\frac{1+\left|e_{1}(t)\right|}{\delta_{1}^{*}}\right)
$$

From Lemmas (5.3) and (5.4) we immediately have 
Lemma 5.5. Vertical descending paths are dissipative for Eq. (4.33) and vertical ascending paths are dissipative for Eq. (4.34) provided the width of the band $S_{a}$ is small enough.

We now consider a path $\gamma(\tau)$ such that

$$
\gamma_{1}(\tau)=\tau, \quad \gamma_{2}(\tau)>0 \text {. }
$$

Lemma 5.6. If the width of the band $S_{a}$ is small enough, there exist paths $\gamma$ satisfying (5.47) which are dissipative for Eq. (4.33) for all $\tau \in \mathbb{R}$ and such that

$$
\lim _{\tau \rightarrow \infty} \gamma_{2}(\tau)=\gamma_{2}(\infty)>0
$$

Proof. From Lemma (5.2) and from (5.31) we must have for $\varphi \in \mathscr{H}_{2}(0)$,

$$
\begin{aligned}
\mathfrak{J}\langle\varphi \mid W(0, \gamma(\tau)) T(\tau) W(\gamma(\tau), 0) \varphi\rangle= & \mathfrak{R}\left\langle\varphi \mid\left(\hat{H}\left(\tau+i \gamma_{2}(\tau)\right)-e_{1}(\tau)\right) \varphi\right\rangle \dot{\gamma}_{2}(\tau) \\
& +\mathfrak{J}\left\langle\varphi \mid\left(\hat{H}\left(\tau+i \gamma_{2}(\tau)\right)-i e_{1}^{\prime}(\tau) \gamma_{2}(\tau)\right) \varphi\right\rangle \leqq 0 .
\end{aligned}
$$

We can choose the width of the band so that the factor in front of $\dot{\gamma}_{2}(\tau)$ is strictly positive (Lemma (5.3)). Thus (5.47) is now equivalent to

$$
\dot{\gamma}_{2}(\tau) \leqq-\frac{\mathfrak{I}\left\langle\varphi \mid\left(\hat{H}\left(\tau+i \gamma_{2}(\tau)\right)-i e_{1}^{\prime}(\tau) \gamma_{2}(\tau)\right) \varphi\right\rangle}{\mathfrak{R}\left\langle\varphi \mid\left(\hat{H}\left(\tau+i \gamma_{2}(\tau)\right)-e_{1}(\tau)\right) \varphi\right\rangle} .
$$

Condition (5.49) is certainly fulfilled for all $\varphi \in \mathscr{H}_{2}(0),\|\varphi\|=1$ if

$$
\dot{\gamma}_{2}(\tau) \leqq-\sup _{\varphi \in \mathscr{H}_{2}(0):\|\varphi\|=1} \frac{\mid \mathfrak{J}\left\langle\varphi\left|\left(\hat{H}\left(\tau+i \gamma_{2}(\tau)\right) \varphi\right\rangle\right|+\left|e_{1}^{\prime}(\tau)\right| \gamma_{2}(\tau)\right.}{\Re\left\langle\varphi \mid\left(\hat{H}\left(\tau+i \gamma_{2}(\tau)\right)-e_{1}(\tau)\right) \varphi\right\rangle}
$$

By Lemma (5.3) we have

$$
\begin{gathered}
\sup _{\varphi \in \mathscr{H}_{2}(0):\|\varphi\|=1} \frac{\mid \mathfrak{I}\left\langle\varphi\left|\left(\hat{H}\left(\tau+i \gamma_{2}(\tau)\right) \varphi\right\rangle\right|+\left|e_{1}^{\prime}(\tau)\right| \gamma_{2}(\tau)\right.}{\mathfrak{R}\left\langle\varphi \mid\left(\hat{H}\left(\tau+i \gamma_{2}(\tau)\right)-e_{1}(\tau)\right) \varphi\right\rangle} \\
\leqq g_{2}(\tau) 2 \gamma_{2}(\tau)+\frac{2\left|e_{1}^{\prime}(\tau)\right|}{e_{2}(\tau)-e_{1}(\tau)} \gamma_{2}(\tau)
\end{gathered}
$$

provided we choose the width of the band so small that for all $\tau \in \mathbb{R}$,

$$
a g_{2}(\tau) \leqq \frac{1}{2}
$$

Therefore it is sufficient to choose $\gamma(\tau)=\tau+i \gamma_{2}(\tau)$ with $\gamma_{2}(\tau)$ solution of the differential equation

$$
\dot{\gamma}_{2}(\tau)=-\gamma_{2}(\tau) 2\left(g_{2}(\tau)+\frac{\left|e_{1}^{\prime}(\tau)\right|}{e_{2}(\tau)-e_{1}(\tau)}\right)
$$

with $\gamma_{2}(0)>0$. Indeed, Eq. (5.53) can be solved explicitly,

$$
\gamma_{2}(\tau)=\gamma_{2}(0) \exp \left(-2 \int_{0}^{\tau}\left(g_{2}(u)+\frac{\left|e_{1}^{\prime}(u)\right|}{e_{2}(u)-e_{1}(u)}\right) d u\right),
$$

and since $\left(g_{2}(u)+\frac{\left|e_{1}^{\prime}(u)\right|}{e_{2}(u)-e_{1}(u)}\right)$ is integrable we can choose $\gamma_{2}(0)$ so that $0<\gamma_{2}(-\infty)<a$ and $\gamma_{2}(\infty)>0$. Therefore condition (5.49) is fulfilled. 
Remarks.

1. It is clear from the above results that we can prove the existence of a dissipative path for Eq. (4.34) so that $\gamma(\tau)=\tau+i \gamma_{2}(\tau)$ with $\gamma_{2}(\tau)<0$ and $\lim _{t \rightarrow \infty} \gamma_{2}(\tau)<0$.

2. The functions $e_{1}(t)$ and $e_{2}(t)$ do not play the same role because we have defined $\lambda(t+i s)=i s e_{1}(t)$. We could work with $\lambda(t+i s)=i s e_{2}(t)$ or with $\lambda(t+i s)=i s \frac{1}{2}\left(e_{1}(t)+e_{2}(t)\right)$ which is more symmetrical.

3. We can remark that the results of this section are valid for unbounded operators. The existence of the dissipative path $\gamma$ of Lemma (5.6) is based only on the estimate of Lemma (5.1). In particular the value of $\gamma_{2}(\infty)$ depends only on $\hat{c}(t), e_{1}(t), e_{2}(t)$ and $\left|e_{1}^{\prime}(t)\right|$. Moreover $\hat{c}(t)$ depends only on the basic estimates of Sect. (2).

\section{Unbounded Operators}

Let $H(z), H^{+}$and $H^{-}$be unbounded operators which satisfy the conditions I, II and III. It is not possible to use the same strategy as before by making the time complex. We prove our main result by approximating the operators by bounded operators. The operators $H(t)$ are uniformly bounded from below for $t \in \mathbb{R}$. Without restricting the generality we suppose in this section that they are bounded from below by 1 for all $t$. We approximate $H(z)$ and $H^{ \pm}$by

$$
H_{n}(z)=n H(z) R(z,-n)
$$

and

$$
H_{n}^{ \pm}=n H^{ \pm} R( \pm,-n)
$$

Lemma 6.1. There exists a constant $a^{\prime}, 0<a^{\prime}<a$, such that the following statements hold

1. For any $n, H_{n}(z)$ is a bounded operator, holomorphic on $S_{a^{\prime}}, H_{n}(z)^{*}=H_{n}(\bar{z})$ and $0 \in \rho\left(H_{n}(z)\right)$.

2. There exists a constant $C^{\prime}$ independent of $n$, such that

$$
\sup _{|s|<a^{\prime}}\left\|\left(H_{n}(t+i s)-H_{n}^{+}\right) \varphi\right\| \leqq \frac{C^{\prime}}{(1+|t|)^{1+\alpha}}\left(\|\varphi\|+\left\|H_{n}^{+} \varphi\right\|\right), \quad t>0
$$

and

$$
\sup _{|s|<a^{\prime}}\left\|\left(H_{n}(t+i s)-H_{n}^{-}\right) \varphi\right\| \leqq \frac{C^{\prime}}{(1+|t|)^{1+\alpha}}\left(\|\varphi\|+\left\|H_{n}^{-} \varphi\right\|\right), \quad t<0 .
$$

3. $H_{n}(z)$ converges strongly to $H(z)$ on $D$, uniformly on $S_{a^{\prime}}$.

4. The operator $U_{n}(t, s)$, solution of

$$
i \varepsilon \frac{\partial}{\partial t} U_{n}(t, s)=H_{n}(t) U_{n}(t, s), \quad U_{n}(s, s)=\mathbb{1}
$$

converges strongly to $U(t, s)$, uniformly in $t$ and $s$ belonging to a compact interval of 
$\mathbb{R}$ and $V_{n}(t, s)$ defined by the equation

$$
i \varepsilon \frac{\partial}{\partial t} V_{n}(t, s)=\left(H_{n}(t)+i \varepsilon\left[P_{1}^{\prime}(t), P_{1}(t)\right]\right) V_{n}(t, s), \quad V_{n}(s, s)=\mathbb{1}
$$

converges strongly, uniformly in $t$ and $s$ belonging to a compact interval of $\mathbb{R}$ to the adiabatic evolution $V(t, s)$.

5. If $H(t)$ satisfies condition $I I I$, then $H_{n}(t)$ satisfies the same conditions for $n$ large enough, and the spectral projectors $P_{1}(t), P_{2}(t)$ are also spectral projectors for $H_{n}(t)$.

Proof.

1. If $-n \in \rho(z)$, then clearly

$$
H_{n}(z)=n \mathbb{1}-n^{2} R(z,-n),
$$

and therefore $H_{n}(z)$ is holomorphic, $H_{n}(z)^{*}=H_{n}(\bar{z})$. If $0 \in \rho(z)$, then $H_{n}(z)^{-1}=$ $\frac{1}{n}(H(z)+n) H(z)^{-1}$. It remains to show that $-n \in \rho(z)$ if $|\mathfrak{I} z|$ is small enough. This follows from (2.18), since for all $n$ and $t \in \mathbb{R}$,

$$
\|R(t,-n)\| \leqq \frac{1}{n+1},
$$

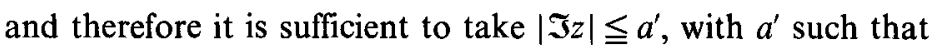

$$
a^{\prime} \sup _{t} c(t) 3=a^{\prime} M^{\prime}<1 \text {. }
$$

2. We have the estimates

$$
\begin{aligned}
\left\|\left(H_{n}(z)-H_{n}^{+}\right) R_{n}(+, 0) \psi\right\| & =\left\|\left(H_{n}(z) R_{n}(+, 0)-\mathbb{1}\right) \psi\right\| \\
& =\left\|\left(H(z) R(z,-n)\left(H^{+}+n\right) R(+, 0)-\mathbb{1}\right) \psi\right\| \\
& =\left\|\left(H(z) R(z,-n)\left(H(z)+n+H^{+}-H(z)\right) R(+, 0)-\mathbb{1}\right) \psi\right\| \\
& =\|(H(z) R(+, 0)+H(z) R(z,-n)(\mathbb{1}-H(z) R(+, 0))-\mathbb{1}) \psi\| \\
& \leqq\left(\sup _{z} \sup _{n}\|H(z) R(z,-n)\|+1\right)\|(H(z) R(+, 0)-\mathbb{1}) \psi\| .
\end{aligned}
$$

For any $z$ we have

$$
\|H(z) R(z,-n)\| \leqq 1+\|n R(z,-n)\|
$$

and from (2.19), with $z=t+i$,

$$
\begin{aligned}
n\|R(z,-n)\| & \leqq n\|R(t,-n)\| \frac{1}{1-a^{\prime} M^{\prime}} \\
& \leqq \frac{1}{1-a^{\prime} M^{\prime}}
\end{aligned}
$$

provided $|s| \leqq a^{\prime}$. Therefore

$$
\left(\sup _{z:|s| \leqq a^{\prime}} \sup _{n}\|H(z) R(z,-n)\|+1\right) \leqq 2+\frac{1}{1-a^{\prime} M^{\prime}}
$$


Considering $\psi=H_{n}^{+} \varphi$, we see from (6.6), (6.9) and condition II that we can take for $C^{\prime}$

$$
C^{\prime}=C\left(2+\frac{1}{1-a^{\prime} M^{\prime}}\right)
$$

where $C$ is the constant of condition II.

3. Let $\psi \in D$. Then

$$
\begin{aligned}
\|n R(z,-n) \psi-\psi\| & =\|R(z,-n) H(z) \psi\| \\
& \leqq\|R(z,-n)\|\|H(z) \psi\| .
\end{aligned}
$$

By condition II, if $z=t+i s$,

$$
\sup _{z:|s| \leqq a^{\prime}}\|H(z) \psi\|<\infty
$$

From (6.11), (6.12) and (6.8) we see that $n R(z,-n)$ converges strongly to the identity on a dense subset $D$, uniformly in $z \in S_{a^{\prime}}$. Since the norm of $n R(z,-n)$ is bounded uniformly in $z \in S_{a^{\prime}}$, we can find, for any $\varphi \in \mathscr{H}$ and any $\varepsilon>0$, a vector $\psi \in D$ such that

$$
\|n R(z,-n) \varphi-\varphi\| \leqq\|(n R(z,-n)-\mathbb{1})(\varphi-\psi)\|+\|(n R(z,-n)-\mathbb{1}) \psi\|
$$

and

$$
\|(n R(z,-n)-\mathbb{1})(\varphi-\psi)\| \leqq \varepsilon \quad \forall n \text { large enough. }
$$

Therefore $n R(z,-n)$ converges strongly to the identity on $\mathscr{H}$, uniformly in $z \in S_{a^{\prime}}$. The map $z \mapsto H(z) \varphi$, for any fixed $\varphi \in D$, is holomorphic and has well-defined limits when $t \rightarrow \pm \infty$ : there exist $\psi^{+}$and $\psi^{-}$such that

$$
\lim _{t \rightarrow \pm \infty} \sup _{|s| \leqq a^{\prime}}\left\|H(t+i s) \varphi-\psi^{ \pm}\right\|=0 .
$$

Consequently the set $\left\{H(z) \varphi ;|\Im z| \leqq a^{\prime}\right\}$ is a compact subset of $\mathscr{H}$ and thus $H_{n}(z) \varphi=n R(z,-n) H(z) \varphi$ converges to $H(z) \varphi$ uniformly on $S_{a^{\prime}}$. Indeed, $n R(z,-n)$ converges strongly to the identity uniformly on any compact subset of $\mathscr{H}$.

4. Let $\varphi(t)$ be a solution of the Schrödinger equation (1.1) with initial condition $\varphi(s)=\varphi^{*} \in D$. We have

$$
i \varepsilon \frac{\partial}{\partial t} \varphi(t)=H_{n}(t) \varphi(t)+\left(H(t)-H_{n}(t)\right) \varphi(t)
$$

and we can write

$$
\varphi(t)=U_{n}(t, s) \varphi^{*}+\int_{s}^{t} U_{n}(t, u)\left(H(u)-H_{n}(u)\right) \varphi(u) d u .
$$

For any fixed $T>|s|$, the function $\left(H(u)-H_{n}(u)\right) \varphi(u)$ converges to zero uniformly on $[-T, T]$. Thus $U_{n}(t, s) \varphi^{*}$ converges uniformly in $t$ and $s \in[-T, T]$ to $U(t, s) \varphi^{*}$. Since $D$ is dense the result follows. The proof of the second statement is the same.

5. The last statement follows easily from the spectral theorem since

$$
H_{n}(t)=f_{n}(H(t)), \quad \text { with } \quad f_{n}(x)=\frac{n x}{x+n} .
$$


Remark. It is essential that the constant $C^{\prime}$ depends only on the constant $C$ of condition II and on $M^{\prime}$ of (6.5), which is independent of $n$.

Proof of Theorem 1.1. Let $A(t)=V^{-1}(t) U(t)$, where $V(t)$ is the adiabatic evolution. We approximate $H$ by $H_{n}$ given in (6.1) and we define $A_{n}(t)=V_{n}^{-1}(t) U_{n}(t)$. The operators $A(t)$, respectively $A_{n}(t)$ are solutions of the equation

$$
A^{\prime}(t)=-V^{-1}(t)\left[P_{1}^{\prime}(t), P_{1}(t)\right] V(t) A(t), \quad A(0)=\mathbb{1},
$$

respectively

$$
A_{n}^{\prime}(t)=-V_{n}^{-1}(t)\left[P_{n, 1}^{\prime}(t), P_{n, 1}(t)\right] V_{n}(t) A_{n}(t), \quad A_{n}(0)=\mathbb{1} .
$$

Assuming that $n$ is large enough we have by Lemma (6.1) that $P_{n, 1}(t)=P_{1}(t)$. Therefore we can assume that

$$
A_{n}^{\prime}(t)=-V_{n}^{-1}(t)\left[P_{1}^{\prime}(t), P_{1}(t)\right] V_{n}(t) A_{n}(t), \quad A_{n}(0)=\mathbb{1} .
$$

With the notation of (3.7) we can write

$$
A(t)=A_{n}(t)+\int_{0}^{t} A_{n}(t, u)\left(K(u)-K_{n}(u)\right) A(u) d u,
$$

where $A_{n}(t, u)$ is solution of (6.21) with $A_{n}(u, u)=\mathbb{1}$. Let $\varepsilon>0$. By Lemma (2.2) there exists $T=T(\varepsilon)$ such that

$$
\int_{-\infty}^{-T}\left\|K(u)-K_{n}(u)\right\| d u+\int_{T}^{\infty}\left\|K(u)-K_{n}(u)\right\| d u \leqq \varepsilon .
$$

By Lemma (6.1) we conclude that $A_{n}(t)$ converges strongly to $A(t)$, uniformly in $t \in \mathbb{R}$, i.e.

$$
\lim _{n \rightarrow \infty} \sup _{t}\left\|\left(A(t)-A_{n}(t)\right) \varphi\right\|=0 .
$$

We must estimate $\mathscr{P}_{21}(\infty,-\infty)$. We have for $n$ large enough by Lemma (6.1), point 5 ,

$$
\begin{aligned}
\left\|P_{2}(0) A(+) A(-)^{-1} P_{1}(0) \varphi\right\| \leqq & \left\|P_{2}(0) A_{n}(+) A_{n}(-)^{-1} P_{1}(0) \varphi\right\| \\
& +\left\|P_{2}(0)\left(A(+) A(-)^{-1}-A_{n}(+) A_{n}(-)^{-1}\right) P_{1}(0) \varphi\right\| .
\end{aligned}
$$

We see that we can prove the theorem by proving it for $H_{n}$, provided that the constant $\kappa=\gamma_{2}(\infty)$ is independent of $n$ and that the constant $M$ of Lemma (4.3) is also independent of $n$ ( $n$ large enough). Indeed the last term disappears as $n \rightarrow \infty$. We already know that $H_{n}$ satisfies conditions I, II and III on $S_{a^{\prime}}$ with constants $\alpha, C^{\prime}$ and $\delta^{*}$ independent of $n$ ( $n$ large enough). We now prove the basic estimate of Lemma (2.1) with an integrable function independent of $n$. The bounded operator $H_{n}(z) H_{n}\left(z^{\prime}\right)^{-1}$ is by definition

$$
\begin{aligned}
H_{n}(z) H_{n}\left(z^{\prime}\right)^{-1} & =H(z) R(z,-n)\left(H\left(z^{\prime}\right)+n\right) R\left(z^{\prime}, 0\right) \\
& =H(z) R(z,-n)\left(H(z)+n+H\left(z^{\prime}\right)-H(z)\right) R\left(z^{\prime}, 0\right) \\
& =H(z) R\left(z^{\prime}, 0\right)+H(z) R(z,-n)\left(H\left(z^{\prime}\right)-H(z)\right) R\left(z^{\prime}, 0\right) .
\end{aligned}
$$


Therefore

$$
\begin{aligned}
\left\|H_{n}(z) H_{n}\left(z^{\prime}\right)^{-1}\right\| \leqq & \left\|H(z) R\left(z^{\prime}, 0\right)\right\| \\
& +\|H(z) R(z,-n)\|\left(1+\left\|H(z) R\left(z^{\prime}, 0\right)\right\|\right) .
\end{aligned}
$$

By (6.9) we have a constant $N_{1}$ independent of $n$ and $z$ such that

$$
\|H(z) R(z,-n)\| \leqq N_{1} .
$$

On the other hand by (2.12) there exists a constant $N_{2}$ such that

$$
\begin{aligned}
\left\|H(z) R\left(z^{\prime}, 0\right) \varphi\right\| & \leqq\left\|R\left(z^{\prime}, 0\right) \varphi\right\|_{z} \\
& \leqq N_{2}\left\|R\left(z^{\prime}, 0\right) \varphi\right\|_{z^{\prime}} \\
& \leqq N_{2}\left(\left\|R\left(z^{\prime}, 0\right)\right\|+1\right)\|\varphi\| .
\end{aligned}
$$

Since $R\left(z^{\prime}, 0\right)$ is a bounded, holomorphic operator admitting $R(+, 0)$ and $R(-, 0)$ as limits at infinity, it is uniformly bounded on $S_{a^{\prime}}$. Therefore there exists a constant $N_{3}$ such that for all $z$ and $z^{\prime}$ in $S_{a^{\prime}}$,

$$
\left\|H_{n}(z) H_{n}\left(z^{\prime}\right)^{-1}\right\| \leqq N_{3},
$$

and thus

$$
\left\|H_{n}(z) \varphi\right\| \leqq N_{3}\left\|H_{n}\left(z^{\prime}\right) \varphi\right\| \text {. }
$$

From this result and Lemma (6.1) we can prove exactly as in Sect. (2) that there exists an integrable function $c(t)$, independent of $n$, such that for all $z=t+i s,|s| \leqq r<a^{\prime}$, all $z^{\prime} \in S_{a^{\prime}}$,

$$
\left\|H_{n}^{\prime}(z) \varphi\right\| \leqq c(t)\left(\|\varphi\|+\left\|H_{n}\left(z^{\prime}\right) \varphi\right\|\right) .
$$

The estimate (6.32) corresponds to Lemma (2.1). For the existence of the dissipative path we need Lemma (5.1). The integrable function $\hat{c}$ must be independent of $n$ and we must prove the estimate of Lemma (2.3) with a constant $N$ independent of $n$. We take in our present case $\lambda=0$. We have from (6.32)

$$
\begin{aligned}
\left\|H_{n}^{\prime}(z) H_{n}(z)^{-1} \varphi\right\| & \leqq c(t)\left(\left\|H_{n}(z)^{-1} \varphi\right\|+\left\|H_{n}(z) H_{n}(z)^{-1} \varphi\right\|\right) \\
& \leqq c(t)\|\varphi\|\left(1+\frac{1}{n}+\|R(z, 0)\|\right) .
\end{aligned}
$$

Therefore there exists a constant $N$ independent of $n$ so that

$$
\left\|H_{n}^{\prime}(z) H_{n}(z)^{-1} \varphi\right\| \leqq N c(t)\|\varphi\| .
$$

From this estimate and Lemma (2.2) we get the existence of an integrable function $\hat{c}(t)$ independent of $n$ such that

$$
\left\|\left(\hat{H}_{n}(t+i s)-\hat{H}_{n}(t)\right) \varphi\right\| \leqq|s| \hat{c}(t)\left(\left\|\hat{H}_{n}(t) \varphi\right\|+\|\varphi\|\right)
$$

(see proof of Lemma (5.1)). Thus there exists a dissipative path $\gamma$ as in Lemma (5.6) which is independent of $n$. It remains to show that the constant $M$ of Lemma (4.3) is also independent of $n$. This is not immediate but the verification of this statement does not present particular difficulties.

Acknowledgements. We thank H. Kunz for interesting discussions. 


\section{References}

1. Arnold, V. I.: Geometrical methods in the theory of ordinary differential equations. 2 nd Ed. Berlin, Heidelberg, New York: Springer 1988

2. Hwang, J.-T., Pechukas, P.: The adiabatic theorem in the complex plane and the semi-classical calculation of non-adiabatic transition amplitudes. J. Chem. Phys. 67, 4640-4653 (1977)

3. Joye, A., Kunz, H., Pfister, Ch.-Ed.: Exponential decay and topological aspects of non-adiabatic transition probabilities. EPFL Preprint (1989)

4. Joye, A., Kunz, H., Pfister, Ch.-Ed.: Exponential decay and geometric aspect of transition probabilities in the adiabatic limit. Ann. Phys. 208, 299-332 (1991)

5. Jaksic, V., Segert, J.: Exponential approach to the adiabatic limit and the Landau-Zener formula. Caltech Preprint (1990)

6. Avron, J. E., Seiler, R., Yaffe, L. G.: Adiabatic theorems and applications to the quantum Hall effect. Commun. Math. Phys. 110, 33-49 (1987)

7. Krein, S. G.: Linear differential equations in Banach spaces. Providence, RI: Transl. Math. Mon. 29, 1971

8. Kato, T.: Perturbation theory for linear operators. Berlin, Heidelberg, New York: Springer 1966

Communicated by J. Fröhlich 